\title{
ALTERAÇÕES FISIOLÓGICAS E DE ERUPÇÃO DENTÁRIA NA OBESIDADE INFANTIL
}

\section{PHYSIOLOGICAL ALTERATION AND DENTAL ERUPTION IN CHILD OBESITY}

\author{
Bárbara Fávero de Araújo Lima** \\ Priscila Hernández de Campos** \\ Renata Gorjão"** \\ Heloisa Helena de Oliveira**** \\ Maria Teresa Botti Rodrigues dos Santos ${ }^{* * * * *}$ \\ Renata de Oliveira Guarét ${ }^{* * \cdots *}$
}

\begin{abstract}
RESUMO
A obesidade infantil vem crescendo e se tornando um problema mundial. Na Odontologia, em relação à obesidade infantil, já foram realizados trabalhos tentando mostrar alguma correlação entre doença cárie e doença periodontal com resultados controversos. Em relação à obesidade infantil e à erupção dentária, alguns trabalhos demostraram evidências de interação, que se apresenta precoce nesses casos, sendo importante observar os trabalhos que avaliam tais correlações. O objetivo desta revisão é associar as relações fisiológicas em crianças obesas e a erupção dentária. Existem alguns fatores que podem justificar a ocorrência dessa erupção precoce como alguns hormônios do sistema endócrino, a leptina e os hormônios sexuais em crianças que apresentam obesidade. Outro ponto é a aceleração na puberdade, concomitantemente com fechamento das epífises e crescimento de ossos longos e da face nessas crianças. A pré-disposição à inflamação crônica também pode influenciar no processo de erupção dentária em crianças obesas. Os profissionais envolvidos no atendimento integral de crianças devem avaliar informações importantes como a troca de dentes e a idade estimada para a erupção dos dentes permanentes, a fim de correlacioná-las, por exemplo, a fatores como a obesidade infantil.
\end{abstract}

Descritores: Erupção dentária • Obesidade pediátrica • Hormônios, Leptina.

\section{ABSTRACT}

Child obesity has been increasing and becoming a worldwide problem. In Odontology, regarding to child obesity, studies have been done trying to show a correlation between dental caries and periodontal disease, but they have had controversial results. In relation to child obesity and dental eruption, studies - that should be referred to - have provided evidence of interaction between them, which appears at early stages in these cases. This revision aims at associating the physiological relations in obese children and dental eruption. There are some factors that can justify the occurrence of these precocious eruptions such as a few hormones from the endocrine system, leptin, and sexual hormones in obese children. Another point is the acceleration in puberty, simultaneously to the closing of the epiphysis and the growth of long bones and of these children's faces. The pre-disposition to chronic inflammation can also influence the process of dental eruption in obese children. The professionals involved in the full care of children must assess important information, such as tooth change and the estimated age for the eruption of permanent teeth, in order to correlate it to factors such as child obesity, for instance.

Descriptors: Tooth eruption • Pediatric obesity • Hormones, Leptin

\footnotetext{
* Cirurgiã-Dentista, graduada pela Universidade Cruzeiro do Sul (Unicsul). Mestre em Ciências da Saúde - Unicsul. Doutoranda do programa de Pós-graduação em Odontopediatria da Unicsul. E-mail: barbara.faverolima@gmail.com

** Cirurgiã-Dentista, graduada pela Unicsul. Mestre em Odontopediatria - Unicsul. Doutoranda do programa de Pós-graduação em Odontopediatria da Unicsul. E-mail: hcampospriscila@yahoo.com.br

*** Graduação em Farmácia e Bioquímica na Usp. Doutora pela Usp. Docente do programa de Pós-graduação Interdisciplinar em Ciências da Saúde da Unicsul. E-mail: renata.gorjao@yahoo.com.br

**** Graduada em farmácia pela Unicsul, doutoranda do Instituto de Ciência da Atividade Física e do Esporte (ICAFE) pela Unicsul. oliveira.helo@hotmail. com

***** Cirurgiã-dentista graduada pela Usp, mestre e doutora pela Unifesp, docente do programa de pós-graduação da Unicsul. E-mail: drsantosmt@yahoo. com.br

****** Cirurgiã-dentista, graduada pela Usp. Mestre e Doutora pela Usp. Docente do programa de Pós-graduação em Odontologia da Unicsul. E-mail: renataguare@uol.com.br
} 


\section{N T R O D U Ç Ã O}

A obesidade pediátrica vem crescendo em muitos países, tornando-se uma preocupação em termos de saúde pública. Em 2013, 42 milhões de crianças (6,3\%) abaixo dos 5 anos estavam com sobrepeso e em 2025 estima-se que $11 \%$ delas adquiram essa condição'.

Os determinantes da obesidade são de caráter demográfico, socioeconômico, epidemiológico, cultural, comportamental, ambiental e psicossocial, o que a torna uma doença multifatorial².

A obesidade é definida como o acúmulo de gordura anormal ou excessivo ${ }^{3,}$ ${ }^{4}$, sendo um distúrbio metabólico caracterizado por estado inflamatório crônico $^{5}$, causado por doenças genéticas, endócrino-metabólicas ou alterações nutricionais ${ }^{6}$.

O aumento do tecido adiposo promove um desequilíbrio imunoendócrino caracterizado pela maior produção de citocinas pró-inflamatórias tais como fator de necrose tumoral alfa (TNF- $\alpha$ ) e a interleucina-6 (IL-6), maior produção de leptina e menor produção de proteínas anti-inflamatórias como a adiponectina ${ }^{7}$. Essas alterações, quando na infância, levam a vários riscos e consequências, como doenças cardiovasculares, dislipidemia, síndrome metabólica, comorbidades pulmonares e gastrointestinais, complicações ortopédicas, problemas psicossociais e neurocognitivos, puberdade precoce, mortalidade ${ }^{8,9,10}$, hipercolesterolemia, hipertensão arterial, síndrome de apneia obstrutiva do sono e comprometimentos osteomioarticulares ${ }^{5}$. A obesidade infantil tem consequências importantes para a morbidade e mortalidade na infância e na vida adulta ${ }^{11,12}$.

Na Odontologia, a relação entre doença cárie e obesidade foi avaliada com resultados controversos. Alguns autores não observaram associação ${ }^{13,14,15,16,17}$ e outros afirmam que essa relação existe ${ }^{18,}$ 19, 20. Quanto à doença periodontal há discordâncias ${ }^{21}$, associadas a fatores inflamatórios, metabólicos, de dieta e higiene ${ }^{22,23}$. Poucos trabalhos avaliaram a relação entre obesidade infantil e erupção dental ${ }^{13,24}$, 25,26 . Todos observaram erupção precoce em crianças obesas e sugerem uma relação com o aumento hormonal e fator de crescimento desses pacientes. Portanto, o objetivo desta revisão é associar as relações fisiológicas em crianças obesas e a erupção dental.

\section{METODOLOGIA}

A busca pelos artigos científicos foi realizada utilizando-se as bases de dados MedLine, SciELO, Bireme, publicados entre os anos de 1980 a 2015. Os descritores utilizados foram: obesidade pediátrica, tecido adiposo, leptina, hormônios, sistema endócrino, erupção dentária. Priorizaram-se as publicações nas línguas inglesa e portuguesa.

\section{REVISÃO DE Literatura E Discussão}

O tecido adiposo era visto como um depósito de ácidos graxos, formador de triacilglicerol, protetor mecânico, isolante térmico e gerador de energia. Contudo, sabe-se que hoje o tecido adiposo tem funções bastante complexas e importantes no funcionamento do organismo humano como, por exemplo, a produção de hormônios ou citocinas ${ }^{27}$.

O sistema endócrino é composto por vários hormônios que regulam diversas áreas do organismo. Em crianças que apresentam obesidade, alguns desses hormônios são mais estudados devido às alterações que podem ocasionar nessa fase, como a leptina, os hormônios tireoidianos T3 (tri-idotironia), T4 (tiroxina), TRH (hormônio de liberação da tireotrofina), TSH (hormônio tireotrófico), GH (hormônio de crescimento) e hormônios sexuais como estrogênio (17- $\beta$-estradiol, estrona e estriol), androgênio, hormônio liberador de gonodotropias (LRF) e hormônios folículo-estimulante $(\mathrm{FSH})$ e luteinizantes ${ }^{4,28}$.

No entanto, o tecido adiposo, quando alterado em tamanho, promove a modificação no padrão de liberação de seus hormônios, as adipocinas. A leptina é uma adipocina que se caracteriza como um componente integral do sistema fisiológico, que sinaliza e modula o estado nutricional e também é um sinal bioquímico que informa o cérebro que as reservas energéticas são suficientes ${ }^{29,30,31}$. O tecido adiposo branco é o principal responsável pela produção de leptina ${ }^{32}$ e a massa total de tecido adiposo é o fator mais associa-
LIMA BFA

CAMPOS PH

GORJÃO R

OLIVEIRA HH

SANTOS MTBR

GUARÉ RO

ALTERAÇÕES

FIS IOLÓGICAS

E DE ERUPÇÃO

DENTÁRIA NA

OBES I DADE

INFANTIL

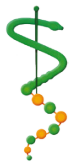

REV. ODONTOL.

UNIV, CID, SÃO

PAULO

$2016 ; 28(1): 50$

7, JAN-ABR 
LIMA BFA

CAMPOS PH

GORJÃO R

OLIVEIRA HH

SANTOS MTBR

GUARÉ RO

ALTERAÇÕES

FISIOLÓGICAS

E DE ERUPÇÃO

DENTÁRIA NA

OBES I DADE

INFANTIL
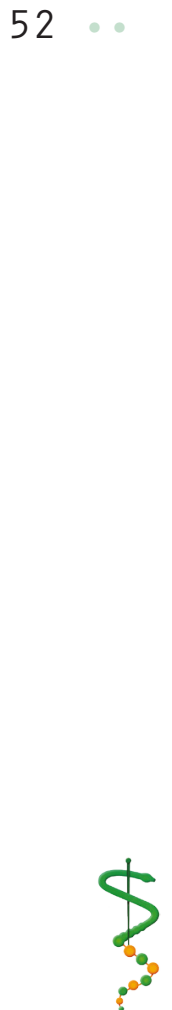

REV, ODONTOL.

UNIV. CID. SÃO

PAULO

2016; 28(1):

$50-7, J A N-A B R$
I SSN 1983-5183

do às concentrações de leptina no sangue. Com isso, o índice de massa corpórea (IMC) está relacionado também à quantidade de leptina circulante no sangue ${ }^{27,28 \text {, }}$ 33,34 .

As oscilações do peso corporal regulam de modo indireto as concentrações de leptina plasmática. A perda do peso corporal provoca a diminuição de leptina plasmática e o aumento, por outro lado, provoca maior concentração da mesma. Assim, essas concentrações de leptina plasmática irão sinalizar o sistema nervoso central (SNC), informando sobre as reservas de energia estocadas em forma de tecido adiposo ${ }^{35}$. Pacientes com obesidade apresentam níveis séricos de leptina proporcionais à sua massa de tecido adiposo e estes apresentam normalmente uma resistência aos efeitos da mesma ${ }^{33,36}$.

Fleisch et al. ${ }^{37}$ (2007) estudaram crianças de 6 a 12 anos recrutadas de 1996 a 2004 que apresentavam alto risco a obesidade por já terem sobrepeso ou que tinham familiares com sobrepeso ou obesidade, para avaliar se os valores séricos de leptina basais apresentavam associação positiva com relação ao aumento do IMC e massa total de gordura. Foram avaliados IMC e massa total de gordura corporal, levando-se em consideração sexo, raça, status socioeconômico, idade, composição corporal, idade óssea e atividade física. Como resultado, observaram que, em condições basais, $43 \%$ das crianças já apresentavam sobrepeso e, ao longo da pesquisa, ocorreu um aumento de $14 \%$ de crianças com sobrepeso, mostrando que a leptina foi estatisticamente significante como preditor para o aumento do IMC e gordura total. Esse aumento da leptina está intimamente associado com o desenvolvimento de outras patologias associadas à desregulação da resposta imune. Em estudo realizado com crianças obesas asmáticas, Youssef et al. ${ }^{38}$ (2013) verificaram que no grupo obeso asmático as crianças apresentaram níveis mais elevados de leptina em relação ao grupo não obeso asmático e ao grupo-controle. Os pesquisadores também encontraram correlação significativa entre os níveis de leptina e de interferon-gama (IFN-gama), uma citocina inflamatória.
Por outro lado, estudos têm demonstrado que a adiponectina é inversamente correlacionada com obesidade, resistência à insulina, risco de desenvolvimento de diabetes, dislipidemia e doenças cardiovasculares ${ }^{39,}{ }^{40}$. Winer et al. ${ }^{41}$ (2006) revelaram, em estudo com 589 crianças e adolescentes obesos, que os baixos níveis de adiponectina estão relacionados com elevada concentração de proteína $C$ reativa (PCR) e triacilgliceróis e baixa concentração de HDL colesterol independente da resistência à insulina e adiposidade. A adiponectina poderia ser um dos sinais que ligam a obesidade à inflamação, podendo ser também um biomarcador para a síndrome metabólica na infância. Essa adipocina atua promovendo uma resposta anti-inflamatória através da inibição da produção da IL-6, interferon-gama (IFN-gama) e indução de interleucina-10 (IL10) em monócitos, macrófagos e linfóci$\operatorname{tos}^{42,43}$

Referente aos hormônios tireoidianos T3 e T4, estes são produzidos pela glândula tireoide e são os principais reguladores do metabolismo somático ${ }^{44,45,46,47}$, atuando na regulação metabólica do sistema alveolar ${ }^{48}$, na manutenção da temperatura corporal, metabolismo de proteínas, lipídeos e vitaminas e na potencialização da ação de outros hormônios ${ }^{49}$. O controle da glândula tireoide envolve mecanismos realizados pelo eixo hipotálamo-hipofisário, hipófise anterior e tireoide. Com isso, o TRH secretado pelo hipotálamo se dirige à adeno-hipófise estimulando a secreção do TSH que age na glândula tireoide estimulando hormônios tireoidianos ${ }^{45 ; 46 ; 48 ; 47 ; 49}$.

Algumas alterações relacionadas aos hormônios tireoidianos como a diabetes melittus, hipotireoidismo, hipertireoidismo, hipoparatireoidismo e hiperparatireoidismo podem levar a alterações bucais. Por exemplo, no hipertireoidismo em crianças, é frequente encontrar uma esfoliação prematura ou acelerada dos dentes decíduos e consequentemente a erupção precoce dos dentes permanentes ${ }^{48}$. $\mathrm{Na}$ literatura, até o momento, não foram encontradas evidências de alterações em relação aos hormônios tireoidianos, mas apresentam, em alguns trabalhos, evidências clínicas da erupção precoce dos ele- 
mentos permanentes, que podem ou não estar relacionados a esses fatores ${ }^{24 ; 13 ; 25 ; 26}$.

A grelina é um hormônio gastrointestinal que também está relacionado ao aumento de tecido adiposo, tendo como uma de suas principais funções a liberação de $\mathrm{GH}^{50}$. O hormônio de crescimento GH exerce um papel importante na regulação do crescimento somático e processos metabólicos ${ }^{51,52,53,54}$ e aumenta a oxidação de ácidos graxos durante a restrição calórica, acelera a lipólise, promove conservação de nitrogênio e alterações da composição corporal. Adultos que apresentam deficiência de GH têm um aumento da massa corporal e redução de massa magra, com importantes consequências no metabolismo lipídico ${ }^{55,56}$. Recentes estudos com roedores sugerem que a grelina, independentemente do $\mathrm{GH}$, diminui a oxidação das gorduras e aumenta a ingestão alimentar e a adiposidade ${ }^{50}$.

Um fator marcante em crianças com deficiência no hormônio GH é a queda progressiva da velocidade de crescimento e retardo de idade óssea. É importante saber que crianças com obesidade podem não responder aos testes de estímulo para $\mathrm{GH}$ e podem ser diagnosticadas erroneamente com essa deficiência. Nesses casos, o diagnóstico diferencial é a idade óssea, que na criança com obesidade estará normal ou avançada, enquanto que em real deficiência, estará retardada ${ }^{57}$.

Em relação aos hormônios sexuais na época da puberdade, ocorrem alterações importantes na sensibilidade no SNC aos estrógenos e eixo hipotálamo-hipófise-gonadal ${ }^{58,59}$. O crescimento longitudinal também ocorre nessa época, com aumento de esteroides sexuais, que induz fusão das epífises e término do crescimento dos ossos longos ${ }^{59,60}$.

Meninas com peso corporal de 20 a $30 \%$ maior que a média, normalmente apresentam menarca mais precoce, demonstrando que a idade da menarca se relaciona com a aquisição de gordura ${ }^{60}$, intimamente ligada à liberação de leptina que é um marcador para o início da puberdade $^{31,61,62}$.

Mesmo que no momento da erupção dos dentes ocorram influências de muitas variáveis, a desordem metabólica é um forte fator importante na precoce esfoliação dos dentes decíduos. Portanto, a ordem e a cronologia de erupção dos dentes permanentes é um acontecimento importante no desenvolvimento dos dentes permanentes e no estabelecimento de uma oclusão correta, difícil de controlar nos dias atuais pelos componentes metabólicos envolvidos ${ }^{63}$.

Alguns trabalhos apresentam relação entre obesidade infantil e erupção dentária, mostrando uma erupção precoce de elementos permanentes em crianças com obesidade $24 ; 13 ; 25 ; 26$. No estudo realizado por Hilgers et al..$^{24}$ (2006), foram avaliadas 104 crianças relacionando IMC, idade e gênero. Como resultado obtiveram que o desenvolvimento dentário foi acelerado de acordo com o IMC, mesmo após o ajuste de gênero e idade. A diferença de cronologia e idade dental foi de $0.68 \pm$ 1.31 anos, sendo que para crianças acima do peso foi de $1.51 \pm 1.22$ anos e crianças obesas de $1.53 \pm 1.28$, mostrando uma diferença estatisticamente significante. Concluíram, portanto, que crianças com sobrepeso e obesidade apresentam um acelerado desenvolvimento dental mesmo após o ajuste de gênero e idade.

No trabalho de Sánchez-Pérez et al. ${ }^{13}$ (2010), foram avaliadas 88 crianças em um estudo longitudinal de 4 anos, e as relações entre doença cárie e IMC e também erupção dentária com IMC. As crianças com IMC alto apresentavam mais dentes erupcionados que as outras, sendo que as com peso normal apresentavam em média 15,9 dentes permanentes e as com sobrepeso 21,6 dentes permanentes, mostrando uma alta relação entre IMC e erupção dental. O mesmo foi visto no estudo de Must et al. ${ }^{26}$ (2012) que também avaliaram IMC e erupção dental e obtiveram como resultado que crianças com obesidade tinham 1,44 mais dentes permanentes que crianças com peso normal $^{26}$. No trabalho de Khan de 2011, que avaliou o tempo de erupção relacionado ao sexo, tipo de escola, altura, peso e IMC de 102 crianças em um estudo longitudinal de um ano, também obteve uma relação positiva entre peso e tempo de erupção dentária ${ }^{25}$
LIMA BFA

CAMPOS PH

GORJÃO R

OLIVEIRA HH

SANTOS MTBR

GUARÉ RO

ALTERAÇÕES

FISIOLÓGICAS

E DE ERUPÇÃO

DENTÁRIA NA

OBESIDADE

INFANTIL

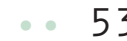

REV. ODONTOL.

Univ. CID. São

PAULO

$2016 ; 28(1): 50$

7 , JAN-ABR 
LIMA BFA

CAMPOS PH

GORJÃO R

OLIVEIRA HH

SANTOS MTBR

GUARÉ RO

ALTERAÇÕES

FISIOLÓGICAS

E DE ERUPÇÃO

DENTÁRIA NA

OBESIDADE

INFANTIL

54

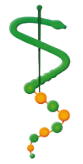

REV, ODONTOL,

UNIV, CID, SÃO PAULO: 2016; 28(1): $50-7, J A N-A B R$

\section{CONCLUSÃO}

Diante desses trabalhos clínicos, há algumas evidências da alteração no tempo de erupção dentária de acordo com o peso corporal mais elevado de crianças, possivelmente relacionado ao aumento da quantidade de leptina e aceleração na puberdade, concomitantemente com fechamento das epífises e crescimento de ossos longos e da face.

Mais estudos são necessários para confirmação dessa hipótese, principalmente entre a relação de hormônios como leptina e alterações bucais, como a erupção dental, já que os hormônios influenciam a maturação óssea. Outro fator importan-

\section{REFERÊNCIAS}

1. Mendis S, Davis S, Norrving B. Organizational update: the world health organization global status report on noncommunicable diseases 2014; one more landmark step in the combat against stroke and vascular disease. Stroke 2015 May;46(5):e121-2.

2. Brasil. Ministério da Saúde. Secretaria de Atenção à Saúde. Departamento de atenção básica. Política nacional de alimentação e nutrição. 2. ed. Brasília: Ministério da Saúde; 2012.

3. Ogden CL, Flegal KM. Changes in terminology for childhood overweight and obesity. Natl Health Stat Report 2010 Jun 25;25):1-5.

4. Souza MCC, Tibúrcio JD, Bicalho JMF, Rennó HMS, Dutra JS, Campos LG, et al. Factors associated with obesity and overweight in school-aged children. Texto contexto - enferm, Florianópolis 2014 Sep;23(3):712-9.

5. Pereira-Lancha LO, Campos-Ferraz PL, Lancha AH. Obesity: considerations about etiology, metabolism, and the use of experimental models. Diabetes, Metabolic Syndrome and Obesity: Targets and Therapy 2012 5(75-87. te são os efeitos que uma predisposição à inflamação crônica podem promover no processo de erupção dentária.

Os profissionais envolvidos no atendimento integral de crianças devem avaliar informações importantes como a troca de dentes e a idade estimada para a erupção dos dentes permanentes, a fim de correlacionar, por exemplo, a fatores como a obesidade infantil.

\section{A GRA DEC I MENTO}

Este estudo foi financiado pelo ConseIho Nacional de Desenvolvimento Científico e Tecnológico (CNPq) sob o protocolo 477040/2012-8.
6. Fisberg M. [Nutritional anemia: prevention or treatment?]. J Pediatr (Rio J) 1995 Mar-Apr;71(2):59-60.

7. Wellen KE, Hotamisligil GS. Inflammation, stress, and diabetes. J Clin Invest 2005 May;115(5):1111-9.

8. Guinhouya BC. Physical activity in the prevention of childhood obesity. Paediatr Perinat Epidemiol 2012 Sep;26(5):438-47.

9. Yanovski JA. Pediatric obesity. An introduction. Appetite 2015 Oct;93(312.

10. Pollock NK. Childhood obesity, bone development, and cardiometabolic risk factors. Mol Cell Endocrinol 2015 Jul 15;410(52-63.

11. Must A, Spadano J, Coakley EH, Field AE, Colditz G, Dietz WH. The disease burden associated with overweight and obesity. JAMA 1999 Oct 27;282(16):1523-9.

12. Lloyd LJ, Langley-Evans SC, McMullen S. Childhood obesity and risk of the adult metabolic syndrome: a systematic review. Int J Obes (Lond) 2012 Jan;36(1):1-11. 
13. Sanchez-Perez L, Irigoyen ME, Zepeda M. Dental caries, tooth eruption timing and obesity: a longitudinal study in a group of Mexican schoolchildren. Acta Odontol Scand 2010 Jan;68(1):57-64.

14. Kopycka-Kedzierawski DT, Auinger P, Billings RJ, Weitzman M. Caries status and overweight in 2- to 18-year-old US children: findings from national surveys. Community Dent Oral Epidemiol 2008 Apr;36(2):157-67.

15. Alves LS, Susin C, Dame-Teixeira N, Maltz M. Overweight and obesity are not associated with dental caries among 12-year-old South Brazilian schoolchildren. Community Dent Oral Epidemiol 2013 Jun;41(3):224-31.

16. Gonzalez Munoz M, Adobes Martin M, Gonzalez de Dios J. [Systematic review about dental caries in children and adolescents with obesity and/or overweight]. Nutr Hosp 2013 Sep-Oct;28(5):1372-83.

17. Hayden C, Bowler JO, Chambers S, Freeman R, Humphris G, Richards D, et al. Obesity and dental caries in children: a systematic review and meta-analysis. Community Dent Oral Epidemiol 2013 Aug;41(4):289-308.

18. Costacurta M, Di Renzo L, Bianchi A, Fabiocchi F, De Lorenzo A, Docimo R. Obesity and dental caries in paediatric patients. A cross-sectional study. Eur J Paediatr Dent 2011 Jun;12(2):112-6.

19. Sakeenabi B, Swamy HS, Mohammed RN. Association between obesity, dental caries and socioeconomic status in 6- and 13-year-old school children. Oral Health Prev Dent 2012 10(3):231-41.

20. Yao $Y$, Ren $X$, Song $X$, He L, Jin $Y$, Chen $Y$, et al. The relationship between dental caries and obesity among primary school children aged 5 to 14 years. Nutr Hosp 2014 30(1):60-5.

21. Katz J, Bimstein E. Pediatric obesity and periodontal disease: a systematic review of the literature. Quintessence Int 2011 Jul-Aug;42(7):595-9.
22. Franchini R, Petri A, Migliario M, Rimondini L. Poor oral hygiene and gingivitis are associated with obesity and overweight status in paediatric subjects. J Clin Periodontol 2011 Nov;38(11):1021-8.

23. Fadel HT, Pliaki A, Gronowitz E, Marild S, Ramberg P, Dahlen G, et al. Clinical and biological indicators of dental caries and periodontal disease in adolescents with or without obesity. Clin Oral Investig 2014 18(2):359-68.

24. Hilgers KK, Akridge M, Scheetz JP, Kinane DE. Childhood obesity and dental development. Pediatr Dent 2006 Jan-Feb;28(1):18-22.

25. Khan N. Eruption Time of Permanent Teeth in Pakistani Children. Iranian Journal of Public Health 2011 40(4):63-73.

26. Must A, Phillips SM, Tybor DJ, Lividini K, Hayes $C$. The association between childhood obesity and tooth eruption. Obesity (Silver Spring) 2012 Oct;20(10):2070-4.

27. Meira TB, Moraes FL, Böhme MTS. Relações entre leptina, puberdade $\mathrm{e}$ exercício no sexo feminino. Rev Bras Med Esporte 2009 Aug.;15(4):306-10.

28. Romero CEM, Zanesco A. O papel dos hormônios leptina e grelina na gênese da obesidade. Rev Nutr 2006 Feb.;19(1):85-91.

29. Flier JS. Clinical review 94: What's in a name? In search of leptin's physiologic role. J Clin Endocrinol Metab 1998 May;83(5):1407-13.

30. Negrão AB, Licinio J. Leptina: o diálogo entre adipócitos e neurônios. Arq Bras Endocrinol Metab 2000 Jun.;44(3):205-14.

31. Cunningham MJ, Clifton DK, Steiner RA. Leptin's actions on the reproductive axis: perspectives and mechanisms. Biol Reprod 1999 Feb;60(2):216-22.
LIMA BFA

CAMPOS PH

GORJÃO R

OLIVEIRA HH

SANTOS MTBR

GUARÉ RO

ALTERAÇÕES

FIS IOLÓGICAS

E DE ERUPÇÃO

DENTÁRIA NA

OBES I DADE

INFANTIL

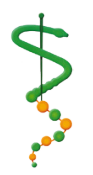

REV, ODONTOL.

UNIV, CID, SÃO

PAULO

$2016 ; 28(1): 50$ -

7 , JAN-ABR 
LIMA BFA

CAMPOS PH

GORJÃO R

OLIVEIRA HH

SANTOS MTBR

GUARÉ RO

ALTERAÇÕES

FISIOLÓGICAS

E DE ERUPÇÃO

DENTÁRIA NA

$O B E S I D A D E$

INFANTIL

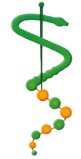

REV, ODONTOL

UNIV, CID, SÃO PAULO

$2016 ; 28(1):$

$50-7$, JAN-ABR
32. Cinti S, Frederich RC, Zingaretti MC, De Matteis R, Flier JS, Lowell BB. Immunohistochemical localization of leptin and uncoupling protein in white and brown adipose tissue. Endocrinology 1997 Feb;138(2):797-804.

33. Considine RV, Sinha MK, Heiman ML, Kriauciunas A, Stephens TW, Nyce $M R$, et al. Serum immunoreactive-leptin concentrations in normal-weight and obese humans. N Eng/ J Med 1996 Feb 1;334(5):292-5.

34. Hassink SG, Sheslow DV, de Lancey E, Opentanova I, Considine RV, Caro JF. Serum leptin in children with obesity: relationship to gender and development. Pediatrics 1996 Aug;98(2 Pt 1):201-3.

35. Benatti FB, Lancha Junior AH. Leptina e exercício físico aeróbio: implicações da adiposidade corporal e insulina. Rev Bras Med Esporte 2007 Aug.;13(4):263-9.

36. Caro JF, Kolaczynski JW, Nyce MR, Ohannesian JP, Opentanova I, Goldman WH, et al. Decreased cerebrospinal-fluid/serum leptin ratio in obesity: a possible mechanism for leptin resistance. Lancet 1996 Jul 20;348(9021):15961.

37. Fleisch AF, Agarwal N, Roberts MD, Han JC, Theim KR, Vexler A, et al. Influence of serum leptin on weight and body fat growth in children at high risk for adult obesity. J Clin Endocrinol Metab 2007 Mar;92(3):948-54.

38. Youssef DM, Elbehidy RM, Shokry DM, Elbehidy EM. The influence of leptin on Th1/Th2 balance in obese children with asthma. J Bras Pneumol 2013 Sep-Oct;39(5):562-8.

39. Panagopoulou P, Galli-Tsinopoulou A, Fleva A, Pavlitou-Tsiontsi E, Vavatsi-Christaki N, Nousia-Arvanitakis S. Adiponectin and insulin resistance in childhood obesity. J Pediatr Gastroenterol Nutr 2008 Sep;47(3):356-62.
40. Jeffery AN, Murphy MJ, Metcalf BS, Hosking J, Voss LD, English $\mathrm{P}$, et al. Adiponectin in childhood. Int J Pediatr Obes 2008 3(3):130-40.

41. Winer JC, Zern TL, Taksali SE, Dziura J, Cali AM, Wollschlager M, et al. Adiponectin in childhood and adolescent obesity and its association with inflammatory markers and components of the metabolic syndrome. J Clin Endocrinol Metab 2006 Nov;91(11):4415-23.

42. Fantuzzi G. Adipose tissue, adipokines, and inflammation. J Allergy Clin Immunol 2005 May;115(5):911-9; quiz 20.

43. Wolf AM, Wolf D, Rumpold H, Enrich $\mathrm{B}$, Tilg $\mathrm{H}$. Adiponectin induces the anti-inflammatory cytokines IL-10 and IL-1RA in human leukocytes. Biochem Biophys Res Commun 2004 Oct 15;323(2):630-5.

44. Bensenor I. Screening for thyroid disorders in asymptomatic adults from Brazilian populations. Sao Paulo Med J 2002 Sep 2;120(5):146-51.

45. Nunes MT. Hormônios tiroideanos: mecanismo de ação e importância biológica. Arq Bras Endocrinol Metab 2003 dez;47(6):639-43.

46. Roberts CG, Ladenson PW. Hypothyroidism. Lancet 2004 Mar 6;363(9411):793-803.

47. Brasil. Agência Nacional de Saúde Suplementar. Primeiras diretrizes clínicas na saúde suplementar: versão preliminar. Rio de Janeiro: ANS; 2009. Disponível em: http://www.ans.gov. br/images/stories/Materiais para pesquisa/Materiais por assunto/ProdEditorialANS Primeiras Diretrizes Clinicas da SS.pdf.

48. Serakides R, Nunes VA, Ocarino NM, Nascimento EF. Efeito da associação hipertireoidismo-castração no osso de ratas adultas. Arq Bras Endocrinol Metab 2004 dez.;48(6):875-84. 
49. Soldá AC. Efeito do laser em baixa intensidade em ratos wistar com hipotireoidismo induzido pelo propiltiouracil (PTU) [Dissertação]. São Paulo: Universidade de São Paulo; 2010. 38 f.

50. Salbe AD, Tschop MH, DelParigi A, Venti CA, Tataranni PA. Negative relationship between fasting plasma ghrelin concentrations and ad libitum food intake. J Clin Endocrinol Metab 2004 Jun;89(6):2951-6.

51. Sherwin RS, Schulman GA, Hendler R, Walesky M, Belous A, Tamborlane W. Effect of growth hormone on oral glucose tolerance and circulating metabolic fuels in man. Diabetologia 1983 Mar;24(3):155-61.

52. Raben MS, Hollenberg $\mathrm{CH}$. Effect of growth hormone on plasma fatty acids. J Clin Invest 1959 38(3):484-8.

53. Davidson MB. Effect of growth hormone on carbohydrate and lipid metabolism. Endocr Rev 1987 May;8(2):11531 .

54. Moller $\mathrm{N}$, Jorgensen JO, Moller J, Orskov L, Ovesen P, Schmitz O, et al. Metabolic effects of growth hormone in humans. Metabolism 1995 Oct;44(10 Suppl 4):33-6.

55. Crist DM, Peake GT, Mackinnon LT, Sibbitt WL, Jr., Kraner JC. Exogenous growth hormone treatment alters body composition and increases natural killer cell activity in women with impaired endogenous growth hormone secretion. Metabolism 1987 Dec;36(12):1115-7.
56. Mauras N, O'Brien KO, Welch S, Rini A, Helgeson K, Vieira NE, et al. Insulin-like growth factor I and growth hormone $(\mathrm{GH})$ treatment in $\mathrm{GH}$-deficient humans: differential effects on protein, glucose, lipid, and calcium metabolism. J Clin Endocrinol Metab 2000 Apr;85(4):1686-94.

57. Damiani D, Damiani D, Oliveira RG. Obesidade: fatores genéticos ou ambientais. Pediatr mod 2002 mar;38(3):57-80.

58. Collaço FM. Endoclinologia: conceitos atuais. Lisboa: Fundação Calouste Gulbenkian 1980.

59. Kase NG, Weingold AB. Ginecologia clínica. Rio de janeiro: Guanabara Koogan; 1987.

60. Peckham BM, Shapiro SS. Sinais e sintomas em ginecologia. 2. ed. São Paulo: Harper e Row do Brasil; 1986.

61. Carro E, Pinilla L, Seoane LM, Considine RV, Aguilar E, Casanueva FF, et al. Influence of endogenous leptin tone on the estrous cycle and luteinizing hormone pulsatility in female rats. Neuroendocrinology 1997 Dec;66(6):375-7.

62. Yura S, Ogawa Y, Sagawa N, Masuzaki $\mathrm{H}$, Itoh $\mathrm{H}$, Ebihara $\mathrm{K}$, et al. Accelerated puberty and late-onset hypothalamic hypogonadism in female transgenic skinny mice overexpressing leptin. J Clin Invest 2000 Mar;105(6):749-55.

63. Feraru I-V, Rãducanu AM, Feraru SE, Herpeliu C. The sequence and chronology of the eruption of permanent canines and premolars in a group of Romanian children in Bucharest. OHDM 2011 Dec;10(4):193-8.

Recebido em 29/04/2016

Aceito em 08/08/2016
LIMA BFA

CAMPOS PH

GORJÃO R

OLIVEIRA HH

SANTOS MTBR

GUARÉ RO

ALTERAÇÕES

FISIOLÓGICAS

E DE ERUPÇÃO

DENTÁRIA NA

OBES I DADE

INFANTIL

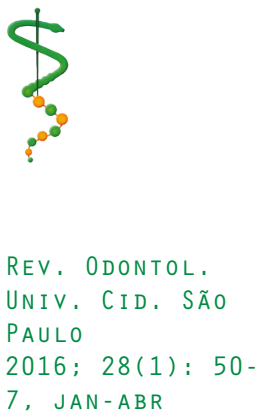

